\title{
Presolar Stardust In The Solar System: Recent Advances for Nuclear Astrophysics
}

\section{Larry R. Nittler*}

Dept. of Terrestrial Magnetism, Carnegie Institution of Washington, 5241 Broad Branch Rd NW,

Washington DC, 20015, USA

E-mail: Inittleraciw.edu

Grains of presolar stardust are identified in extraterrestrial materials on the basis of highly anomalous isotopic compositions. These compositions reflect those of the stellar gases from which they condensed more than 4.5 billion years ago and serve as detailed probes of nuclear processes in space including Galactic chemical evolution, nucleosynthesis and mixing processes in stars. A large number of presolar phases have now been identified, including various oxides, silicates, carbides and elemental carbon, with apparent origins in red giants, asymptotic giant branch stars, supernovae and perhaps novae. This paper provides some illustrative examples of how presolar stardust provides new insights for nuclear astrophysics.

10th Symposium on Nuclei in the Cosmos

July 27 - August 12008

Mackinac Island, Michigan, USA

\footnotetext{
*Speaker.
} 


\section{Introduction}

Presolar stardust grains are tiny dust specks that condensed in outflows and explosions of previous generations of stars and survived processing in the interstellar medium and early Solar System $[1,2,3]$. They comprise a minor portion $(<\mathrm{ppb}$ to a few hundred $\mathrm{ppm})$ of primitive meteorites and interplanetary dust particles (microscopic extraterrestrial samples collected in the stratosphere). They were discovered in 1987, following a long search for carriers of isotopically anomalous noble gases in meteorites. Because they escaped homogenization processes in the early solar system, they are identified by their highly unusual isotopic compositions, relative to all other materials in the solar system. These compositions reflect both Galactic chemical evolution (GCE) and nuclear processing in the parent stars. Because the presolar grains can be studied in great detail in modern microanalytical laboratories, they can provide high-precision constraints on nuclear astrophysics, complementary to traditional astronomical observations.

Each presolar grain is a sample of a specific place in a specific star at a specific time, with very little or no processing since its formation. Presolar stardust can thus provide important information about a range of astrophysical processes. In particular, the ability to precisely determine the isotopic composition of multiple elements in a single presolar grain places unprecedented quantitative constraints on stellar evolution and nucleosynthesis models (e.g. [4]). Mineralogical and microstructural studies provide detailed information on grain formation processes in stars (e.g., $[5,6])$. A detailed review of presolar grains and their applications in astrophysics and space science is beyond the scope of this paper. Here I will focus on some examples and recent advances to illustrate how meteoritic stardust provides new insights and quantitative constraints on nuclear processes in the galaxy. The interested reader is referred to longer reviews (e.g., [1, 2, 3]) and the current literature for additional information.

\section{Analysis and Types of Presolar Grains}

The discovery and increasingly detailed characterization of presolar grains has been made possible by technological advances in micro- and nano-analytical instrumentation. For example, modern secondary ion mass spectrometers (SIMS) can determine, with high sensitivity, isotopic ratios of many elements in sub-micrometer solid samples, allowing for identification of presolar grains with astrophysically relevant sizes $[7,8,9]$. Once identified, a presolar grain can be further analyzed by a range of techniques. For example, additional isotopic signatures might be determined using SIMS, noble-gas mass spectrometry [10], or resonance ionization mass spectrometry (RIMS, $[11,12])$. Detailed chemical and mineralogical investigations can be carried out by scanning and transmission electron microscopy and/or Auger spectroscopy (e.g., [5, 13, 6, 14]).

Example images of a few of the known types of presolar grains are shown in Fig. 1; some basic information for the known presolar phases are summarized in Table 1. Most of the carbonaceous grains (and $\mathrm{Si}_{3} \mathrm{~N}_{4}$ ) have been identified in acid residues of meteorites from which the dominant phases (silicates, metal, sulfides) have been removed. O-rich phases have been identified primarily by automated techniques originally in acid residues [15, 16], but now also in situ $[7,8,9]$ via isotopic imaging. Some phases (refractory carbides, metal) have been identified as sub-grains within larger presolar graphite grains $[5,13,17]$. Note that although several hundred 


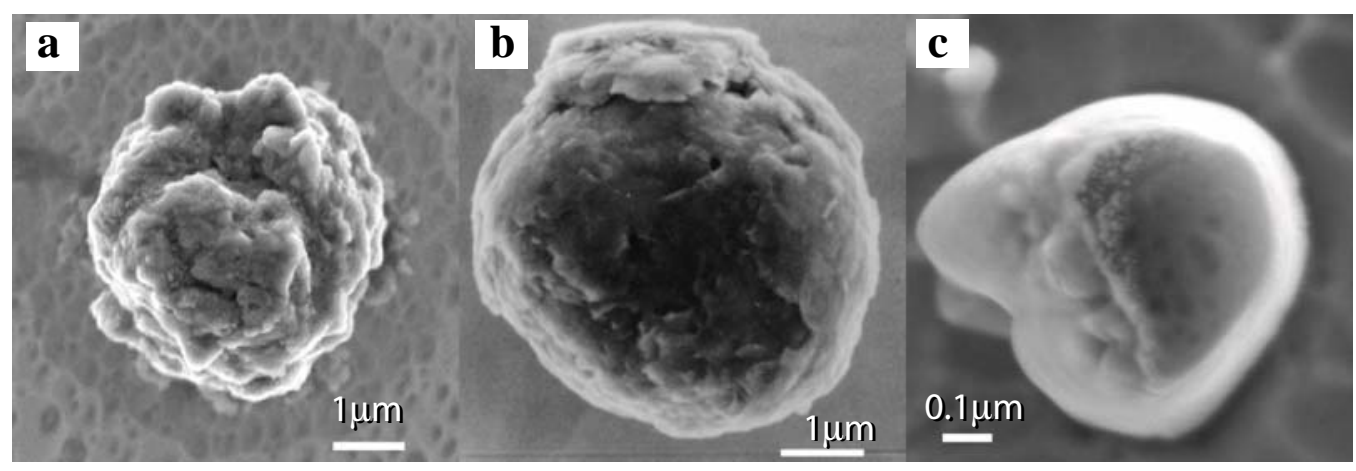

Figure 1: Electron micrographs of presolar grains: a) SiC; b) Graphite; c) $\mathrm{Al}_{2} \mathrm{O}_{3}$; Based on [3].

Table 1: Types of presolar grains in meteorites and interplanetary dust particles (IDPs), after [2]. $\mathrm{AGB}=$ Asymptotic Giant Branch stars, $\mathrm{SNe}=$ Supernovae, $\mathrm{RG}=$ Red Giant stars.

\begin{tabular}{|c|c|c|c|}
\hline Phase & $\begin{array}{l}\text { Abundance } \\
(\mathrm{ppm})\end{array}$ & Size & Source(s) \\
\hline Nanodiamond & 1400 & $2 \mathrm{~nm}$ & $\mathrm{SNe}(?)$ \\
\hline $\begin{array}{l}\text { Silicates (olivine, pyroxene, } \\
\text { Ca-, Al-rich, glass ...) }\end{array}$ & $\begin{array}{l}\approx 500 \text { (IDPs) } \\
\approx 100 \text { (meteorites) }\end{array}$ & $0.1-1 \mu \mathrm{m}$ & $\mathrm{AGB}, \mathrm{SNe}$ \\
\hline $\mathrm{SiC}$ & 10 & $0.1-20 \mu \mathrm{m}$ & AGB, SNe, J-stars, novae(?) \\
\hline Graphite & $1-2$ & $1-20 \mu \mathrm{m}$ & $\mathrm{AGB}, \mathrm{SNe}$ \\
\hline TiC, $\mathrm{ZrC}, \mathrm{MoC}, \mathrm{RuC}, \mathrm{FeC}, \mathrm{Fe}-\mathrm{Ni}$ & (sub-grains in graphite) & $5-220 \mathrm{~nm}$ & $\mathrm{AGB}, \mathrm{SNe}$ \\
\hline Silicon Nitride $\left(\mathrm{Si}_{3} \mathrm{~N}_{4}\right)$ & $>0.002$ & $\sim 1 \mu \mathrm{m}$ & $\mathrm{SNe}$ \\
\hline $\begin{array}{l}\text { Oxides }\left(\mathrm{Al}_{2} \mathrm{O}_{3}, \mathrm{MgAl}_{2} \mathrm{O}_{4},\right. \\
\left.\qquad \mathrm{CaAl}_{12} \mathrm{O}_{19}, \mathrm{TiO}_{2},(\mathrm{Mg}, \mathrm{Fe}) \mathrm{Cr}_{2} \mathrm{O}_{4}\right)\end{array}$ & $>10$ & $0.1-3 \mu \mathrm{m}$ & $\mathrm{RG}, \mathrm{AGB}, \mathrm{SNe}$ \\
\hline
\end{tabular}

presolar silicate grains have now been identified, very few have had detailed mineralogical identifications. Chemical analysis indicates a wide range of silicate compositions. Moreover, more than half that have been analyzed by transmission electron microscopy have proven to be amorphous, non-stoichiometric phases. Note also that the origin for the meteoritic nanodiamonds is unsettled; a solar system origin for most of the diamonds cannot be excluded [18].

\section{Implications for Nuclear Astrophysics}

Because the parent stars of presolar grains ended their lives more than $4.5 \mathrm{Gyr}$ ago, one must use an iterative approach to identify the type of star that produced any given grain. For example, Fig. 2 shows the distributions of $\mathrm{C}$ and $\mathrm{N}$ isotopic ratios measured in presolar $\mathrm{SiC}$ grains. The data fall into distinct groupings. Comparison with spectroscopic observations and theoretical models indicates that these families represent different types of stellar sources, as indicated on the Figure. For example, the $\mathrm{C}$ isotopic distribution of the dominant "Mainstream" population is remarkably similar to that observed in C-rich asymptotic giant branch (AGB) stars [19], pointing to these as sources. Many other isotope signatures in the grains point to an AGB source as well and the highprecision data obtainable on the grains can thus be used to constrain AGB models. Similarly, the "X-grains" have signatures pointing to an origin in Type II supernovae and can provide unique information about such explosions of massive stars. Analogous groupings of O isotopic ratios of 


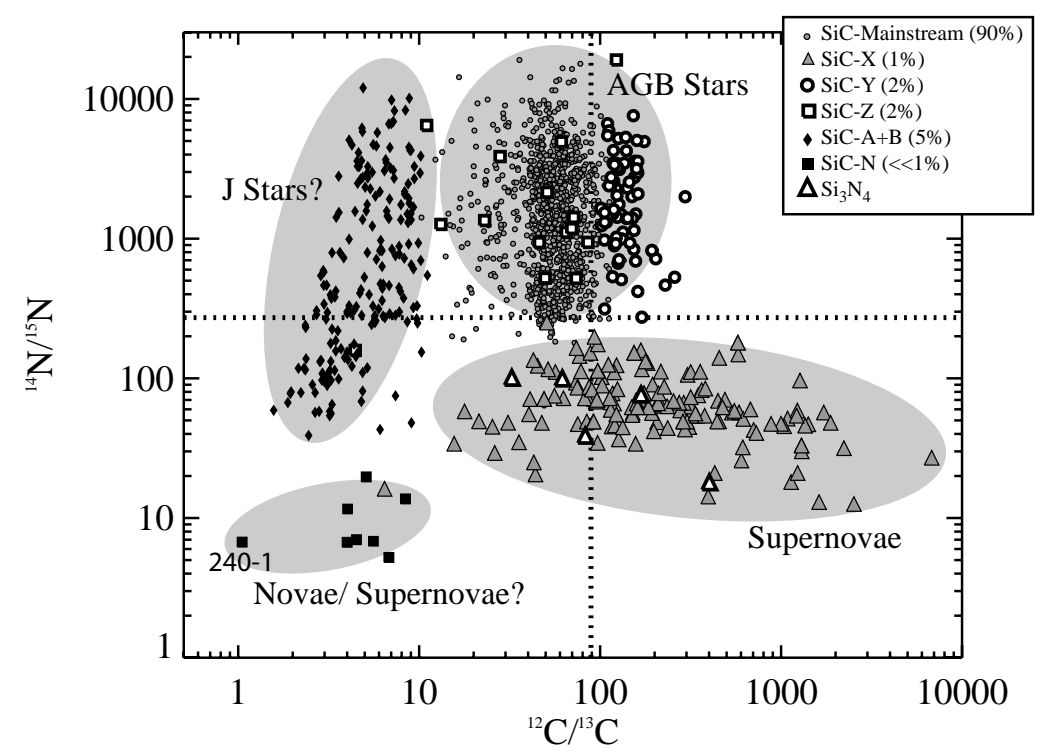

Figure 2: $\mathrm{C}$ and $\mathrm{N}$ isotopic ratios measured in individual presolar $\mathrm{SiC}$ grains (see [2] for data sources). Different groupings of isotopic compositions can be attributed to different stellar sources, as indicated. Grain 240-1 [20] is the best candidate yet identified for a nova condensate. Dotted lines indicate solar ratios in this and subsequent figures.

presolar oxide and silicate grains have been used to identify red giants, AGB stars and SNe as the sources of these (Fig. 3).

\subsection{Grains from Low-mass Stars}

It is now well accepted that most $\mathrm{SiC}$ grains originated in low-mass $\left(<2 \mathrm{M}_{\odot}\right)$ AGB stars: the mainstream grains from roughly solar-metallicity stars, and the rare $\mathrm{Y}$ and $\mathrm{Z}$ grains from lowermetallicity stars. Among the strongest pieces of evidence for this comes from the isotopic composition of heavy trace elements in single grains, made possible by resonance ionization mass spectrometry (RIMS). RIMS measurements have revealed almost-pure $s$-process isotopic signatures of many elements, including $\mathrm{Mo}, \mathrm{Zr}, \mathrm{Sr}, \mathrm{Ba}$, and $\mathrm{Ru}$ (e.g., [4]). In fact, the relatively high precision of the measurements provides for quantitative constraints on $s$-process models. For example, a recent comparison of models with data acquired for multiple elements in single SiC grains [21] constrains the amount of ${ }^{13} \mathrm{C}$ present in the region between the AGB He- and H-burning shells, a free parameter in AGB nucleosynthesis calculations [22, 4], to a narrow range around that required to explain the solar $s$-process abundance distribution. Moreover, deviations between model trends and $\mathrm{SiC}$ trace-element data have pointed both to the original presence of radioactive elements [12] and to incorrect neutron-capture cross-sections [23, 24].

Most presolar O-rich grains (Group 1, Fig. 3a) are enriched in ${ }^{17} \mathrm{O}$ and slightly depleted in ${ }^{18} \mathrm{O}$, relative to solar composition. Their compositions are well-explained by models of dredge-up in low-mass red giant stars, provided they formed from stars with a range of masses and metallicities [15]. The $\mathrm{O}$ isotope distribution of these and the Group 3 grains strongly indicates the existence of an age-metallicity relation in the presolar solar neighborhood of the Galaxy. Initial ${ }^{26} \mathrm{Al} /{ }^{27} \mathrm{Al}$ and 

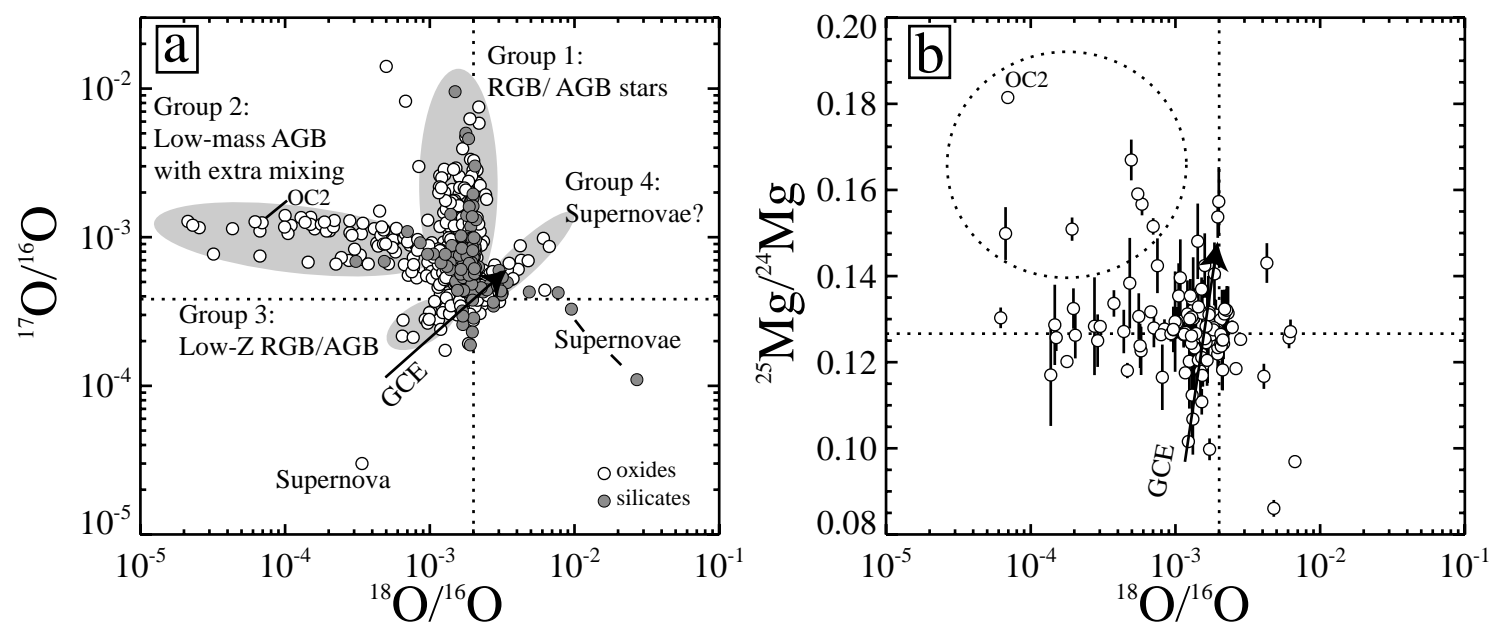

Figure 3: $O$ isotopic ratios measured in individual presolar oxide and silicate grains (see $[2,16]$ for data sources). Different grain Groups and individual grains can be attributed to different stellar sources, as indicated. Arrows indicate rough trends expected for GCE of isotopic ratios; dotted ellipse in (b) indicates unusually ${ }^{25} \mathrm{Mg}$-rich and ${ }^{18} \mathrm{O}$-poor grains.

${ }^{41} \mathrm{Ca} /{ }^{40} \mathrm{Ca}$ ratios, inferred for many of the grains from excesses in radioactive daughter products, are in good agreement with predictions for low-mass AGB stars [16]. The more ${ }^{18} \mathrm{O}$-depleted Group 2 grains likely formed in low-mass AGB stars experiencing "extra" mixing, not predicted by standard 1-d models of stellar evolution. Extra mixing is also indicated by high inferred initial ${ }^{26} \mathrm{Al} /{ }^{27} \mathrm{Al}$ ratios in the Group 2 grains as well as by some $\mathrm{C}$ an $\mathrm{N}$ isotopic compositions of presolar $\mathrm{SiC}$ grains $[25,26,27]$. The physical mechanism of the mixing (often called "cool bottom processing" or CBP, [28]) is unknown, but may be related to shear instabilities, thermohaline instabilities and/or magnetic effects $[29,30,31]$. The high-precision grain data can help constrain parameters of mixing models, for example the mixing rate and temperature reached by circulating material [26]. Interestingly, the mixing parameters required to explain the $\mathrm{SiC}$ data and the oxide data appear to be quite different, indicating that CBP operates differently in O-rich versus C-rich AGB stars.

Mg isotopes are affected by both GCE [32] and nuclear processing in stars [33] and Mg is one of the few elements amenable to spectroscopic isotopic analysis in stars [34]. Recent high-precision $\mathrm{Mg}$ isotopic data for presolar oxide grains (Fig. 3b) are thus valuable for comparisons with observations and model predictions $[35,36,16]$. Group 1 grains show evidence for the expected increase in ${ }^{25} \mathrm{Mg} /{ }^{24} \mathrm{Mg}$ with metallicity due to GCE, though there is considerable scatter. Several ${ }^{18} \mathrm{O}$-depleted Group 2 grains show relatively large excesses in ${ }^{25} \mathrm{Mg}$ (dotted ellipse in Fig. 3b), comparable to those predicted for intermediate-mass AGB (IM-AGB) stars. However, the O isotopic ratios of these grains indicate lower mass stellar parents, which are not predicted to have such ${ }^{25} \mathrm{Mg}$ excesses. An IM-AGB origin suggested for the extreme grain OC2 [36] has also been ruled out by recent re-evaluation of the ${ }^{16} \mathrm{O}(\mathrm{p}, \gamma){ }^{17} \mathrm{~F}$ reaction rate [37]. The unusual ${ }^{25} \mathrm{Mg}$ excesses observed in these grains as well as many main-sequence stars might indicate strongly heterogeneous GCE of $\mathrm{Mg}$ isotopes or perhaps mass-transfer from IM-AGB companions to lower-mass stars in binary systems [16]. 


\subsection{Grains from Supernovae and Novae}

A small fraction of presolar $\mathrm{SiC}$ ("X grains"), the very rare $\mathrm{Si}_{3} \mathrm{~N}_{4}$ grains and a larger fraction of presolar graphite grains are believed to originate in the cooling ejecta of Type II supernovae $(\mathrm{SN})$. The strongest evidence for a $\mathrm{SN}$ origin is the observation of large ${ }^{44} \mathrm{Ca}$ excesses, unaccompanied by anomalies in other stable Ca isotopes, in many grains $[38,39]$. This signature points to in situ decay of ${ }^{44} \mathrm{Ti}$, which has a half-life of $\approx 60 \mathrm{y}$ and is produced solely in SN. Moreover, extinct ${ }^{49} \mathrm{~V}$ observed in some grains [40] also indicates a supernova origin and requires that they formed within one year of the parental explosions. A large number of additional isotope signatures observed in the grains also point to a SN origin. A key observation of the supernova grain data is the apparent necessity to heterogeneously mix material from different zones in order to quantitatively reproduce grain isotopic compositions using detailed SN nucleosynthesis calculations [39, 41, 42]. Although extensive macroscopic mixing is both observed in and predicted for SN ejecta, the detailed microscopic mixing required by the grains poses challenges to our understanding of $\mathrm{SNe}$ (e.g., [43]).

Isotopic data for the SN grains have both provided important insights into supernova nucleosynthesis and raised important puzzles. For example, the isotopic patterns observed for heavy elements $\mathrm{Mo}, \mathrm{Zr}, \mathrm{Sr}, \mathrm{Ba}$ and $\mathrm{Fe}$ in $\mathrm{SiC} \mathrm{X}$ grains are completely distinct from those observed in the AGB grains, and also differ from expectations for $r$ - or $p$-processes associated with supernovae. Rather, these point to a "neutron-burst" nuclear process occurring in a massive star's $\mathrm{He}$ shell during the explosion [44]. A key unsolved problem is the fact that the grains are uniformly more ${ }^{15} \mathrm{~N}$-rich than can be explained by SN mixing models (Fig. 4). Qualitatively, the data point to mixing of the He- and C-rich layer (enriched in ${ }^{12} \mathrm{C}$ and ${ }^{15} \mathrm{~N}$ ) and the overlying $\mathrm{He} / \mathrm{N}$ zone, to explain high ${ }^{26} \mathrm{Al} /{ }^{27} \mathrm{Al}$ ratios. However, the extremely high abundance of ${ }^{14} \mathrm{~N}$ in the $\mathrm{He} / \mathrm{N}$ zone has precluded quantitative matching of the data (mixing curves in Fig. 4). The problem is exacerbated by the recent identification of supernova $\mathrm{SiC}$ and graphite grains with very low ${ }^{12} \mathrm{C} /{ }^{13} \mathrm{C}$ ratios ( $\lesssim 10,[45,46])$; these data point to the existence a $\mathrm{SN}$ reservoir enriched in ${ }^{13} \mathrm{C},{ }^{15} \mathrm{~N}$ and ${ }^{26} \mathrm{Al}$. Such a reservoir is hinted at in the most recent SN models of Heger and colleagues (A. Heger, pers. comm.). In their models of stars of mass $\approx 25-30 \mathrm{M}_{\odot}$, a small region of the $\mathrm{He} / \mathrm{N}$ zone is enriched in ${ }^{15} \mathrm{~N}$, relative to the zone as a whole (grey ellipses in fig. 4). The nucleosynthetic origin of this ${ }^{15} \mathrm{~N}$ "spike" is as-yet unresolved, but may be due to hot CNO burning during the SN explosion. In any case, even this new region is not sufficiently rich in ${ }^{15} \mathrm{~N}$ to explain the data (dashed mixing curves in Fig. 4) but it points in the right direction. An alternative explanation, extensive production of ${ }^{15} \mathrm{~N}$ during core He-burning in rotating massive stars [47], deserves further investigation as well. Finally, unusual $\mathrm{Fe}, \mathrm{Ni}, \mathrm{Ca}$ and $\mathrm{Ti}$ isotopic compositions were recently reported in presolar SN grains $[46,48]$. It remains to be seen whether these can be adequately explained by nucleosynthesis models or if they will point to new insights about massive star evolution.

A small fraction of presolar oxide and silicate grains also likely formed in $\mathrm{SNe}$ (Fig. 3a). Interestingly, although the most abundant product of Type II SNe is ${ }^{16} \mathrm{O}$, only one ${ }^{16} \mathrm{O}$-rich $\mathrm{SN}$ grain has been found [49]. The remainder of O-rich grains believed to have a $\mathrm{SN}$ origin are ${ }^{18} \mathrm{O}$ rich $[50,16]$, reflecting a contribution from the He-burning zone of the massive star. A SN origin for many of these grains is supported by ${ }^{25} \mathrm{Mg}$ depletions, due to partial mixing of ${ }^{24} \mathrm{Mg}$-rich material from the inner parts of the parent SN (Fig. 3b, [16]). That a majority of presolar SN O-rich grains 


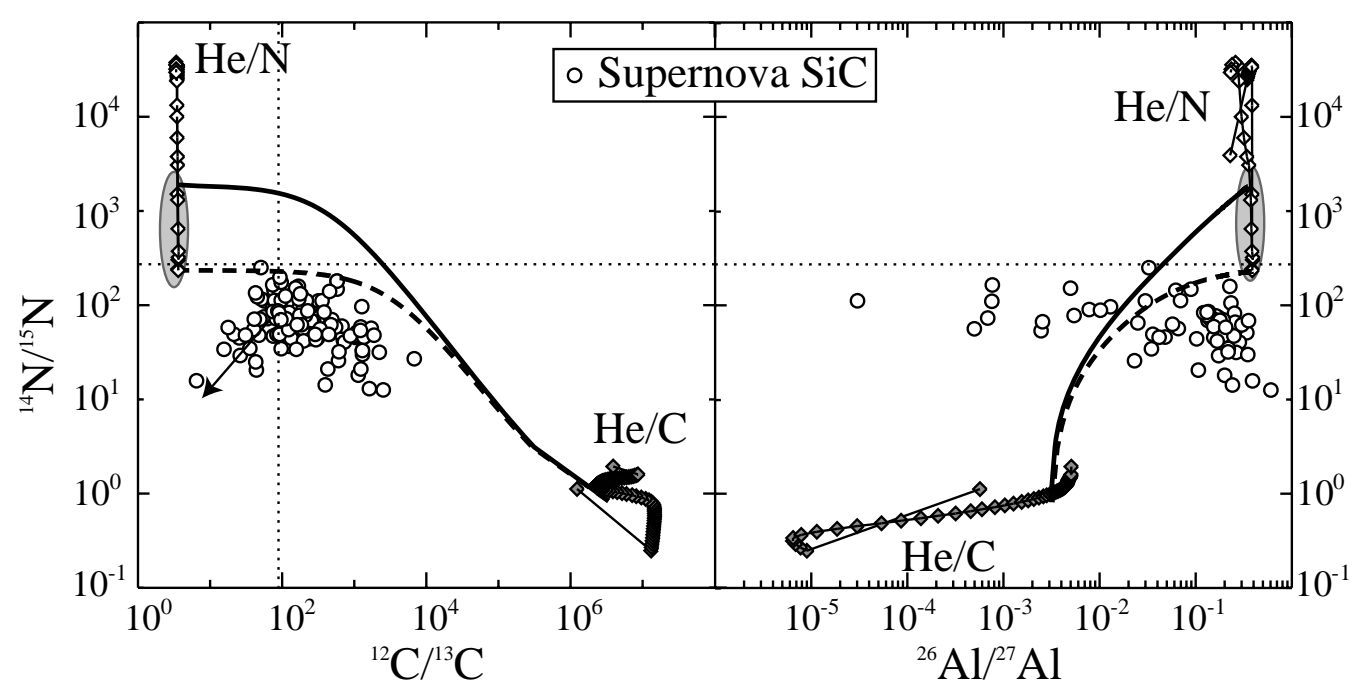

Figure 4: Isotopic ratios measured in individual presolar $\mathrm{SiC} \mathrm{X}$-grains from supernovae compared with predicted compositions of two zones of a $25 \mathrm{M}_{\odot}$ supernova (diamonds, Heger \& Woosley, pers. comm.). A ${ }^{15} \mathrm{~N}$ rich sub-region of the He/N zone, not seen in previous $\mathrm{SN}$ calculations, is indicated by grey ellipses. Solid curves indicate mixing between the average compositions of $\mathrm{He} / \mathrm{N}$ and $\mathrm{He} / \mathrm{C}$ zones; dashed curves indicate mixing between the $\mathrm{He} / \mathrm{C}$ zone and the most ${ }^{15} \mathrm{~N}$-rich sub-region of $\mathrm{He} / \mathrm{N}$ zone. Arrow in left panel indicates data trend towards highly ${ }^{13} \mathrm{C}$ - and ${ }^{15} \mathrm{~N}$-rich composition.

are apparently from outer layers of the parent stars might reflect preferential destruction of dust grains in deeper layers by reverse shocks [51].

Amari et al. [52] proposed a nova origin for a handful of isotopically unusual $\mathrm{SiC}$ and graphite grains, based mostly on very low ${ }^{12} \mathrm{C} /{ }^{13} \mathrm{C}$ and ${ }^{14} \mathrm{~N} /{ }^{15} \mathrm{~N}$ ratios (Fig. 2), but also on ${ }^{30} \mathrm{Si}$ enrichments. However, a nova origin for these grains is somewhat problematic because the pure nova nucleosynthetic signatures must be greatly diluted to explain the data. Moreover, the identification of a supernova $\mathrm{SiC}$ grain with closely similar $\mathrm{C}$ and $\mathrm{N}$ isotopes [45] shows that $\mathrm{SNe}$ can produce compositions with lower ${ }^{12} \mathrm{C} /{ }^{13} \mathrm{C}$ and ${ }^{14} \mathrm{~N} /{ }^{15} \mathrm{~N}$ ratios than predicted and calls into question whether the other grains formed in novae or SNe. More recently, an Al-rich SiC grain was identified (240-1, Fig. 2, [20]) with ${ }^{12} \mathrm{C} /{ }^{13} \mathrm{C}=1$, low ${ }^{14} \mathrm{~N} /{ }^{15} \mathrm{~N}$ and high inferred ${ }^{26} \mathrm{Al} /{ }^{27} \mathrm{Al}$. This grain's composition agrees well with nova models [53], without any dilution and is the strongest candidate found to date for a nova condensate in a meteorite.

\section{Acknowledgments:}

I thank Ernst Zinner, Stan Woosley, Alex Heger and Conel Alexander for helpful discussions. I am very grateful to Drs. Woosley and Heger for sharing detailed unpublished supernova nucleosnythesis data.

\section{References}

[1] L. R. Nittler, Presolar stardust in meteorites: recent advances and scientific frontiers, Earth Planet. Sci. Lett. 209 (2003) 259-273.

[2] E. Zinner, Presolar grains, in Treatise on Geochemistry vol 1: Meteorites, Comets, and Planets (A. M. Davis, ed.), pp. 17-39, 2004 (updated 2007). 
[3] D. D. Clayton and L. R. Nittler, Astrophysics with presolar stardust, Ann. Rev. Astron. Astrophys. 42 (2004) 39-78.

[4] M. Lugaro, A. M. Davis, R. Gallino, M. J. Pellin, O. Straniero, and F. Käppeler, Isotopic compositions of strontium, zirconium, molybdenum, and barium in single presolar SiC grains and asymptotic giant branch stars, Astrophys. J. 593 (2003) 486-508.

[5] T. J. Bernatowicz, R. Cowsik, P. C. Gibbons, K. Lodders, J. Fegley, Bruce, S. Amari, and R. S. Lewis, Constraints on stellar grain formation from presolar graphite in the Murchison meteorite, Astrophys. J. 472 (1996) 760-782.

[6] R. M. Stroud, L. R. Nittler, and C. M. O’D. Alexander, Polymorphism in presolar $\mathrm{Al}_{2} \mathrm{O}_{3}$ grains from asymptotic giant branch stars, Science 305 (2004) 1455-1457.

[7] S. Messenger, L. P. Keller, F. J. Stadermann, R. M. Walker, and E. Zinner, Samples of stars beyond the Solar System: Silicate grains in interplanetary dust, Science 300 (2003) 105-108.

[8] A. N. Nguyen and E. Zinner, Discovery of ancient silicate stardust in a meteorite, Science 303 (2004) 1496-1499.

[9] K. Nagashima, A. Krot, and H. Yurimoto, Stardust silicates from primitive meteorites, Nature 428 (2004) 921-924.

[10] P. R. Heck, K. K. Marhas, P. Hoppe, R. Gallino, H. Baur, and R. Wieler, Presolar He and Ne isotopes in single circumstellar SiC grains, Astrophys. J. 656 (2007) 1208-1222.

[11] G. K. Nicolussi, A. M. Davis, M. J. Pellin, R. S. Lewis, R. N. Clayton, and S. Amari, s-process zirconium in presolar silicon carbide grains, Science 277 (1997) 1281-1283.

[12] M. R. Savina, A. M. Davis, C. E. Tripa, M. J. Pellin, R. Gallino, R. S. Lewis, and S. Amari, Extinct technetium in silicon carbide stardust grains: Implications for stellar nucleosynthesis, Science $\mathbf{3 0 3}$ (2004) 649-652.

[13] T. K. Croat, T. Bernatowicz, S. Amari, S. Messenger, and F. J. Stadermann, Structural, chemical, and isotopic microanalytical investigations of graphite from supernovae, Geochim. Cosmochim. Acta 67 (2003) 4705-4725.

[14] C. Floss, F. J. Stadermann, J. P. Bradley, Z. R. Dai, S. Bajt, G. Graham, and A. S. Lea, Identification of isotopically primitive interplanetary dust particles: A NanoSIMS isotopic imaging study, Geochim. Cosmochim. Acta 70 (2006) 2371-2399.

[15] L. R. Nittler, C. M. O’D. Alexander, X. Gao, R. M. Walker, and E. Zinner, Stellar sapphires: The properties and origins of presolar $\mathrm{Al}_{2} \mathrm{O}_{3}$ in meteorites, Astrophys. J. 483 (1997) 475-495.

[16] L. R. Nittler, C. M. O’D. Alexander, R. Gallino, P. Hoppe, A. Nguyen, F. Stadermann, and E. K. Zinner, Aluminum-, calcium- and titanium rich oxide stardust in ordinary chondrite meteorites, Astrophys. J. 682 (2008) 1450-1478.

[17] T. K. Croat, F. J. Stadermann, and T. J. Bernatowicz, Presolar graphite from AGB stars: Microstructure and s-process enrichment, Astrophys. J. 631 (2005) 976-987.

[18] Z. R. Dai, J. P. Bradley, D. J. Joswiak, D. E. Brownlee, H. G. M. Hill, and M. J. Genge, Possible in situ formation of meteoritic nanodiamonds in the early Solar System, Nature 418 (2002) 157-159.

[19] D. L. Lambert, B. Gustafsson, K. Eriksson, and K. H. Hinkle, The chemical composition of carbon stars. I. carbon, nitrogen, and oxygen in 30 cool carbon stars in the galactic disk., Astrophys. J. Supp. 62 (1986) 373-425. 
[20] L. R. Nittler, C. M. O’D. Alexander, and A. N. Nguyen, Extreme ${ }^{13} C$ and ${ }^{15} N$ enrichments in a Murchison presolar SiC grain, Meteoritics and Plan. Sci. 41 (Supp) (2006) Abstract \#5316.

[21] J. G. Barzyk, M. R. Savina, A. M. Davis, R. Gallino, F. Gyngard, S. Amari, E. Zinner, M. J. Pellin, R. S. Lewis, and R. N. Clayton, Constraining the C-13 neutron source in AGB stars through isotopic analysis of trace elements in presolar SiC, Meteoritics and Plan. Sci. 42 (2007) 1103-1119.

[22] R. Gallino, C. Arlandini, M. Busso, M. Lugaro, C. Travaglio, O. Straniero, A. Chieffi, and M. Limongi, Evolution and nucleosynthesis in low-mass asymptotic giant branch stars. II. neutron capture and the s-process, Astrophys. J. 497 (1998) 388-403.

[23] F. Voss, K. Wisshak, K. Guber, F. Käppeler, and G. Reffo, Stellar neutron capture cross sections of the Ba isotopes, Phys.Rev. C 50 (1994) 2582-2601.

[24] P. Koehler, J. A. Harvey, and K. H. G. D. Wiarda, High-resolution neutron capture and total cross-section measurements, and the astrophysical ${ }^{95} \operatorname{Mo}(n, \gamma)$ reaction rate at s-process temperatures, this volume.

[25] C. M. O'D. Alexander and L. R. Nittler, The galactic evolution of Si, Ti, and O isotopic ratios, Astrophys. J. 519 (1999) 222-235.

[26] K. M. Nollett, M. Busso, and G. J. Wasserburg, Cool bottom processes on the thermally pulsing asymptotic giant branch and the isotopic composition of circumstellar dust grains, Astrophys. J. 582 (2003) 1036-1058.

[27] E. Zinner, L. R. Nittler, R. Gallino, A. I. Karakas, M. Lugaro, O. Straniero, and J. C. Lattanzio, Silicon and carbon isotopic ratios in AGB stars: SiC grain data, models, and the Galactic evolution of the Si isotopes, Astrophys. J. 650 (2006) 350-373.

[28] G. J. Wasserburg, A. I. Boothroyd, and I.-J. Sackmann, Deep circulation in red giant stars: A solution to the carbon and oxygen isotope puzzles?, Astrophys. J. 447 (1995) L37-L40.

[29] J. P. Zahn, Circulation and turbulence in rotating stars, Astron. Astrophys. 265 (1992) 115-132.

[30] P. P. Eggleton, D. S. P. Dearborn, and J. C. Lattanzio, Deep mixing of ${ }^{3}$ He: Reconciling Big Bang and stellar nucleosynthesis, Science 314 (2006) 1580-1583, [arXiv : astro-ph/0611039].

[31] J. Nordhaus, M. Busso, G. J. Wasserburg, E. G. Blackman, and S. Palmerini, Magnetic mixing in red giant and asymptotic giant branch stars, Astrophys. J. 684 (2008) L29-L32.

[32] Y. Fenner, B. K. Gibson, H.-c. Lee, A. I. Karakas, J. C. Lattanzio, A. Chieffi, M. Limongi, and D. Yong, The chemical evolution of magnesium isotopic abundances in the solar neighbourhood, Publications of the Astronomical Society of Australia 20 (2003) 340-344.

[33] A. I. Karakas, M. A. Lugaro, M. Wiescher, J. Görres, and C. Ugalde, The uncertainties in the ${ }^{22} \mathrm{Ne}+\alpha$-capture reaction rates and the production of the heavy magnesium isotopes in asymptotic giant branch stars of intermediate mass, Astrophys. J. 643 (2006) 471-483, [arXiv:astro-ph/0601645].

[34] D. Yong, D. L. Lambert, and I. I. Ivans, Magnesium isotopic abundance ratios in cool stars, Astrophys. J. 599 (2003) 1357-1371, [arXiv:astro-ph/0309079].

[35] E. Zinner, L. R. Nittler, P. Hoppe, R. Gallino, O. Straniero, and C. M. O’D. Alexander, Oxygen, magnesium and chromium isotopic ratios of presolar spinel grains, Geochim. Cosmochim. Acta 69 (2004) 4149-4165. 
[36] M. Lugaro, A. I. Karakas, L. R. Nittler, C. M. O’D. Alexander, P. Hoppe, C. Iliadis, and J. C. Lattanzio, On the asymptotic giant branch star origin of peculiar spinel grain OC2, Astron. Astrophys. 461 (2007) 657-664, [arXiv:astro-ph/0610464].

[37] C. Iliadis, C. Angulo, P. Descouvemont, M. Lugaro, and P. Mohr, New reaction rate for O16 $(p, \gamma) F 17$ and its influence on the oxygen isotopic ratios in massive AGB stars, Phys.Rev. C 77 (2008) 045802, [arXiv:0803.2757].

[38] L. R. Nittler, S. Amari, E. Zinner, S. E. Woosley, and R. S. Lewis, Extinct ${ }^{44}$ Ti in presolar graphite and SiC: proof of a supernova origin, Astrophys. J. 462 (1996) L31-L34.

[39] P. Hoppe, R. Strebel, P. Eberhardt, S. Amari, and R. S. Lewis, Isotopic properties of silicon carbide X grains from the Murchison meteorite in the size range 0.5-1.5 $\mu \mathrm{m}$, Meteoritics and Plan. Sci. 35 (2000) 1157-1176.

[40] P. Hoppe and A. Besmehn, Evidence for extinct vanadium-49 in presolar silicon carbide grains from supernovae, Astrophys. J. 576 (2002) L69-L72.

[41] C. Travaglio, R. Gallino, S. Amari, E. Zinner, S. Woosley, and R. S. Lewis, Low-density graphite grains and mixing in Type II supernovae, Astrophys. J. 510 (1999) 325-354.

[42] T. Yoshida, Supernova mixtures reproducing isotopic ratios of presolar grains, Astrophys. J. 666 (2007) 1048-1068.

[43] E.-A. Deneault, D. D. Clayton, and A. Heger, Supernova reverse shocks and SiC growth, Astrophys. J. 594 (2003) 312-325.

[44] B. S. Meyer, D. D. Clayton, and L.-S. The, Molybdenum and zirconium isotopes from a supernova neutron burst, Astrophys. J. $\mathbf{5 4 0}$ (2000) L49-L52.

[45] L. R. Nittler and P. Hoppe, Are presolar silicon carbide grains from novae actually from supernovae?, Astrophys. J. 631 (2005) L89-L92.

[46] M. Jadhav, S. Amari, K. K. Marhas, E. Zinner, T. Maruoka, and R. Gallino, New stellar sources for high-density, presolar graphite grains, Astrophys. J. 682 (2008) 1479-1485.

[47] N. Langer, A. Heger, S. E. Woosley, and F. Herwig, Nucleosynthesis in rotating stars, in Nuclei in the Cosmos V (N. Prantzos, ed.), pp. 129-135. Editions Frontiéres, 1998.

[48] K. K. Marhas, S. Amari, F. Gyngard, E. Zinner, and R. Gallino, Iron and nickel isotopic ratios in presolar SiC grains, Astrophys. J. in press.

[49] L. R. Nittler, C. M. O’D. Alexander, J. Wang, and X. Gao, Meteoritic oxide grain from supernova found, Nature 393 (1998) 222.

[50] S. Messenger, L. P. Keller, and D. S. Lauretta, Supernova olivine from cometary dust, Science 309 (2005) 737-741.

[51] T. Nozawa, T. Kozasa, A. Habe, E. Dwek, H. Umeda, N. Tominaga, K. Maeda, and K. Nomoto, Evolution of dust in primordial supernova remnants: Can dust grains formed in the ejecta survive and be injected into the early interstellar medium?, Astrophys. J. 666 (2007) 955-966, [arXiv:0706.0383].

[52] S. Amari, X. Gao, L. R. Nittler, E. Zinner, J. José, M. Hernanz, and R. S. Lewis, Presolar grains from novae, Astrophys. J. 551 (2001) 1065-1072.

[53] J. José, M. Hernanz, S. Amari, K. Lodders, and E. Zinner, The imprint of nova nucleosynthesis in presolar grains, Astrophys. J. 612 (2004) 414-428. 